\title{
Biot number effect and non-Fourier effect on temperature field and stress intensity factor of a cracked strip under thermal shock loading
}

\author{
Wei $\mathrm{LI}^{\mathrm{a}}$, Jia $\mathrm{LI}^{\mathrm{c}}$, Fan Song ${ }^{\mathrm{b}, *}$ \\ ${ }^{a}$ School of Mechanical and Electrical Engineering, North China Institute of Aerospace Engineering, Langfang, PR China \\ ${ }^{\mathrm{b}}$ State Key Laboratory of Nonlinear Mechanics, Institute of Mechanics, Chinese Academy of Sciences, Beijing, PR China \\ ${ }^{\mathrm{c}}$ LSPM, CNRS UPR 3407, Université Paris XIII, Villetaneuse, France
}

\section{A R T I C L E I N F O}

\section{Keywords:}

Biot's number

Non-Fourier effect

Crack

Fourier transform

Singular integral equations

\begin{abstract}
A B S T R A C T
In this paper, we considered a thermal shock problem of a strip with an inner crack parallel to the heated or cooled surface. The effect of the surface heat transfer properties and the non-Fourier effect, which are modified by effective Biot's number and the non-Fourier factor, were considered to investigate the transient temperature field and the dynamic stress intensity factors (SIFs). Fourier transform and Laplace transform were used to reduce this mixed boundary value problem to singular integral equations (SIEs). The Gauss-Chebyshev numerical integration method was applied to the SIEs and linear equations are obtained. Finally, numerical inverse Laplace transform was used to obtain the temperature field and SIFs. The numerical results showed that Biot's number not only affects the uniformity of the temperature field and the magnitude of the SIFs strongly, but also makes contributions to the non-Fourier effect. This finding can help better understanding the fracture behaviors of advanced materials and making a progress to determine the applicable conditions of the non-Fourier effect.
\end{abstract}

\section{Introduction}

The thermal shock problem is an important issue in improving the performance of advanced materials and high-temperature engineering structures, such as thermal barrier materials [1-9]. To solve this problem, the temperature field should be determined first. When the classical Fourier thermal conduction theory is applied on large temperature gradient, the assumption that thermal disturbance will be felt instantaneously at distances infinitely far from its source becomes unacceptable [10]. Researchers began to pay attention to this paradox science around 1950. Cattaneo [11,12] and Vernotte [13] modified the classical Fourier model by introducing thermal relaxation time to the thermal conduction equation. The modified non-Fourier model, known as the hyperbolic heat conduction model or the $\mathrm{C}-\mathrm{V}$ model, results in a hyperbolic heat conduction equation and a finite heat wave propagation speed. Joseph et al [14] proposed the Jefferey's type model for heat conduction, which would predict a smoother heat wave front. Özişik and Tzou [15-17] claimed that the relaxation of both heat flux and temperature gradient should be taken into account, which leads to the dual phase lagging model. Alvarez and Jou [18] elucidated that the in nanosystems, the phonon wave package can be assumed to a series of standing waves with the wavelength the fraction of characteristic size of systems.

\footnotetext{
*Corresponding author at: State Key Laboratory of Nonlinear Mechanics(LNM), Institute of Mechanics, Chinese Academy of Sciences, No. 15 Beisihuanxi Road, Beijing 100190, PR China.

E-mail address: songf@lnm.imech.ac.cn (F. Song).
} 
In recent years, a great amount of thermal elastic study of cracked materials has been carried out based on C-V model. Chen and $\mathrm{Hu}[19,20]$ studied the temperature and SIF behavior of a cracked plate under thermal shock loading with considering the nonFourier effect. Guo et al [21] studied the similar problem with a model of a cracked cylinder. Atkinson et al [22] and Fu et al [23] introduced the coupled effect of the thermos-elasticity to the cylinder model and pointed out that taking the thermomechanical coupling effect into account does not bring too much difference in comparison with the uncoupled model. Moreover, Li et al [24] and $\mathrm{Li}$ [25] comprehensively studied the thermal barrier effect of the crack, the non-Fourier effect and the inertia effect in his research. The authors pointed out that the non-Fourier effect was not negligible when the study scale is very small or in some special materials, such as some non-homogeneous materials [26] or biological tissue materials [27], while the inertia effect was only considerable in small scale [25]. However, the thermal barrier effect of the crack, which is related to the heat conduction performance of crack gap, was significant to the distribution of both temperature and stress field in both small and large scales. In addition, the magnitude of the thermal barrier effect was influenced by many factors, such as the material properties, the filler of the crack gap, the temperature and so on. Therefore, the thermal barrier effect study would help better understanding the failure mechanisms of the materials and provide guidance for the design of new materials.

Apart from all these factors, another important factor in failure analysis of thermal shock is the surface heat transfer coefficient, which may depend on many factors such as the thermal properties of the solid and the liquid, their temperatures at thermal shock, the surface state of the solid etc. A high surface heat transfer performance leads to a high temperature gradient in the solid near the surface, and therefore to high thermal stresses. Basing on hollow cylinder model, Guo [21,28] and Fu [10] found the Biot's numbers of the surfaces affect the temperature fields and the SIFs remarkably. However, the heat transfer study of the heated and free surfaces of the crack problem is rare.

In this article, the surface heat transfer effect and non-Fourier effect on a cracked plate under thermal shock loading were studied in a same framework. Newton's law of cooling which describes the heat flux to or from a surface by convection heat transfer was applied to the entire boundary, including the crack surfaces. Three heat transfer coefficients, which correspond to three different effective Biot's numbers in dimensionless form, were examined in this research. In addition, the hyperbolic heat conduction theory was adopted to analyze the thermal fracture characteristics of an isotropic plate containing a crack. By solving mixed boundary problem, the effect of effective Biot's number and the non-Fourier effect on the thermal fracture response was analyzed.

\section{Statement of the problem and basic equations}

We consider a strip which contains a straight crack of half-length $r$ parallel to the free surface, as shown in Fig. 1 .

The material is assumed to be homogeneous, isotropic and linearly elastic. A Cartesian coordinate system is established with the $x$ axis coincided with the crack plane and the origin at the crack center. The distance between the crack and the two boundaries are $l_{a}$ and $l_{b}$, respectively, as shown in Fig. 1 . In this work, only thermal loading is considered in evaluating the temperature and stress fields. The initial temperature is assumed to be uniform and equals to $T_{0}$ in the plate. Then the free surface $y=-l_{a}$ is suddenly exposed to a convective-medium of temperature $T_{\infty}$, while the ambient temperature in the range of $y>l_{b}$ stays the initial temperature $T_{0}$. Convective heat transfer conditions are applied at all the boundaries and surfaces.

\subsection{Temperature fields}

The famous Fourier's law of heat conduction presents a linear relationship between the heat flux $\vec{q}$ through a material and the gradient of temperature $T$, as $\vec{q}=-k \nabla T$, where $k$ is the material thermal conductivity and $\nabla$ is the gradient operator. In principle, however, the Fourier's law leads to an unphysical infinite heat propagation speed within a continuum for transient heat conduction processes because of its parabolic characteristics. To overcome this contradiction, a hyperbolic model, named the Cattaneo-Vernotte model, has been proposed by introducing a time-dependent term into the Fourier equation [26,29]. The introduced time-derivative term describes a wavy nature of heat propagation at a finite speed, which has been proved in both theory and experiments [29-31]. The governing equation of hyperbolic thermal conduction writes

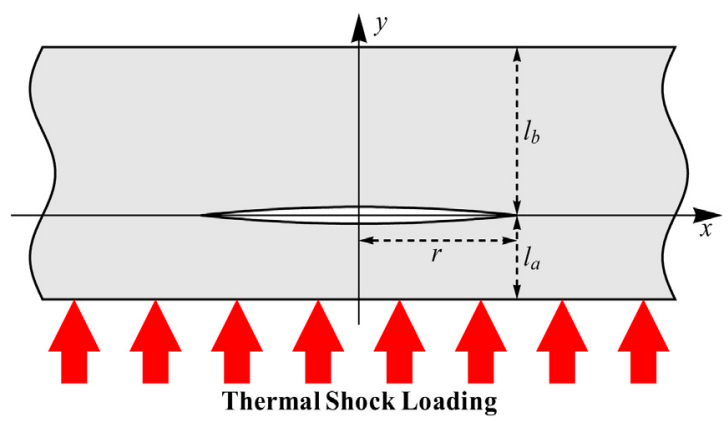

Fig. 1. A finite strip with an inner crack and initial temperature $T_{0}$ suddenly exposed to a convective medium of temperature $T_{\infty}$. 


$$
\nabla^{2} T_{i}=\frac{1}{a}\left(\frac{\partial T_{i}}{\partial t}+\tau_{0} \frac{\partial^{2} T_{i}}{\partial t^{2}}\right), \quad i=1,2
$$

where the subscript 1 and 2 of $T$ denote the temperature in the range of $-l_{a}<y<0$ and $0<y<l_{b}$, respectively; $a=k /\left(\rho C_{p}\right)$ stands for thermal diffusivity; $t$ is the time; $\rho, C_{p}$ and $\tau_{0}$ are the mass density, the specific heat capacity the thermal relaxation time, respectively. Referring to Fig. 1, the hyperbolic heat Eq. (1) is subjected to the following boundary conditions:

$$
\left\{\begin{array}{lrl}
k \frac{\partial T_{1}}{\partial y}=h_{1}\left(T_{1}-T_{\infty}\right) & |x|<\infty, y=-l_{a}, t>0 & (a) \\
k \frac{\partial T_{2}}{\partial y}=h_{2}\left(T_{0}-T_{2}\right) & |x|<\infty, y=l_{b}, t>0 & (b) \\
k \frac{\partial T_{1}}{\partial y}=k \frac{\partial T_{2}}{\partial y}=h_{3}\left(T_{2}-T_{1}\right) & |x|<r, y=0, t>0 & (c) \\
k \frac{\partial T_{1}}{\partial y}=k \frac{\partial T_{2}}{\partial y} & |x| \geqslant r, y=0, t>0 & (d) \\
T_{1}=T_{2} & |x| \geqslant r, y=0, t>0 & (e)
\end{array}\right.
$$

where $h_{i}(i=1,2,3)$, in unit $\mathrm{W} /\left(\mathrm{m}^{2} \cdot \mathrm{K}\right)$, denote the heat transfer coefficient of the lower surface, the upper surface and the crack surface, respectively. In the boundary conditions (2), equations $(a)-(c)$ denote heat is transferred by convection in all the free surfaces; Equations $(d)$ and $(e)$ denote the heat flow and temperature are continuous at the extension line of the crack. The initial conditions of the thermal conduction problem are:

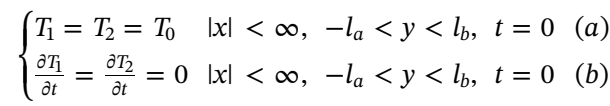

\subsection{Thermo-elastic field equations}

The two-dimensional thermo-elastic theory provides formulations involving the solution of boundary-value problems. In this class of problems two special cases, namely the plane stress and the plane strain should be distinguished. In the frame of the plane elasticity theory, the solution of a plane stress problem can be converted to that of the corresponding plane strain problem and vice versa. The basic equations of the 2-dimension thermos-elasticity problem for a homogeneous isotropic body are:

(1) Equilibrium equations in absence of the body forces:

$$
\left\{\begin{array}{l}
\frac{\partial \sigma_{x}}{\partial x}+\frac{\partial \tau_{x y}}{\partial y}=0 \\
\frac{\partial \tau_{x y}}{\partial x}+\frac{\partial \sigma_{y}}{\partial y}=0
\end{array}\right.
$$

(2) Hooke's law:

$$
\left\{\begin{array}{c}
\sigma_{x}=\frac{\mu}{\kappa-1}\left[(1+\kappa) \frac{\partial u}{\partial x}+(3-\kappa) \frac{\partial v}{\partial y}-4 \alpha\left(T-T_{0}\right)\right] \\
\sigma_{y}=\frac{\mu}{\kappa-1}\left[(3-\kappa) \frac{\partial u}{\partial x}+(1+\kappa) \frac{\partial v}{\partial y}-4 \alpha\left(T-T_{0}\right)\right] \\
\tau_{x y}=\mu\left(\frac{\partial u}{\partial y}+\frac{\partial v}{\partial x}\right)
\end{array}\right.
$$

where $u$ and $v$ are the displacement components; $\varepsilon_{x}, \varepsilon_{x}, \varepsilon_{x y}$, are the components of the strain tensor; $\mu$ and $\alpha$ are the shear modulus and the coefficient of linear thermal expansion, respectively, $\kappa=3-4 \nu$ for plane strain and $\kappa=(3-\nu) /(1+\nu)$ for generalized plane stress. By substituting the stress-displacement relationships (5) into the equilibrium equations (4) we obtain:

$$
\left\{\begin{array}{l}
(1+\kappa) \frac{\partial^{2} u}{\partial x^{2}}+(\kappa-1) \frac{\partial^{2} u}{\partial y^{2}}+2 \frac{\partial^{2} v}{\partial x \partial y}=4 \alpha \frac{\partial T}{\partial x} \\
(\kappa-1) \frac{\partial^{2} v}{\partial x^{2}}+(1+\kappa) \frac{\partial^{2} v}{\partial y^{2}}+2 \frac{\partial^{2} u}{\partial x \partial y}=4 \alpha \frac{\partial T}{\partial y}
\end{array}\right.
$$

The thermal shock problem should be solved under the following boundary conditions and initial conditions: 


$$
\left\{\begin{array}{llll}
\sigma_{y 1}=0 & |x|<\infty, & y=-l_{a} & (a) \\
\tau_{x y 1}=0 & |x|<\infty, & y=-l_{a} & (b) \\
\sigma_{y 2}=0 & |x|<\infty, & y=l_{b} & (c) \\
\tau_{x y 2}=0 & |x|<\infty, & y=l_{b} & (d) \\
\sigma_{y 1}=\sigma_{y 2} & |x|>r, & y=0 & (e) \\
\tau_{x y 1}=\tau_{x y 2} & |x|>r, & y=0 & (f) \\
\sigma_{y 1}=\sigma_{y 2}=0 & |x| \leqslant r, & y=0 & (g) \\
\tau_{x y 1}=\tau_{x y 2}=0 & |x| \leqslant r, & y=0 & (h) \\
u_{1}=u_{2} & |x|>r, & y=0 & (i) \\
v_{1}=v_{2} & |x|>r, & y=0 & (j)
\end{array}\right.
$$

where the subscript 1 and 2 of the displacements and stresses denote the temperature in the range of $-l_{a}<y<0$ and $0<y<l_{b}$, respectively. In (7), $(a)-(d)$ translate the traction free conditions at the top and bottom surfaces of the plate; $(e)$ and $(f)$ denote the stress continuity across the ligaments which extend the crack while $(g)$ and $(h)$ describe the traction free conditions of the crack surfaces; $(i)$ and $(j)$ denote the displacements are continuous at the extension line of the crack. The initial conditions are written as follows:

$$
\left\{\begin{array}{lll}
u_{i}=v_{i}=0 & |x|<\infty,-l_{a}<y<l_{b}, & t=0 \quad(a) \\
\sigma_{x i}=\sigma_{y i}=\tau_{x y i}=0 & |x|<\infty,-l_{a}<y<l_{b}, & t=0
\end{array}\right.
$$

\section{Solving process}

In the following, we present the resolution procedure of the above-defined thermo-elastic equations. Globally speaking, the Laplace and the Fourier transform techniques and Gauss-Chebyshev numerical integration method are used in resolving the problem.

\subsection{Temperature fields}

As is discussed previously, the governing equations of the temperature fields and the displacements in the form of partial differential equations with the mixed boundary conditions are obtained. Obviously, the solution of the temperature fields and displacement fields can be written as:

$$
\left\{\begin{array}{c}
T=T\left(x, y, t, a, k, \tau_{0}, h_{1}, h_{2}, h_{3}, r, l_{a}, l_{b}\right) \\
u=u\left(x, y, t, a, k, \tau_{0}, \kappa, \mu, \alpha, h_{1}, h_{2}, h_{3}, r, l_{a}, l_{b}\right) \\
v=v\left(x, y, t, a, k, \tau_{0}, \kappa, \mu, \alpha, h_{1}, h_{2}, h_{3}, r, l_{a}, l_{b}\right)
\end{array}\right.
$$

The form of solutions contains the coordinate variables $\times, y$; the time variable $t$; the material parameters $a, k, \tau_{0}, h_{1}, h_{2}, h_{3}, \alpha, \mu, \kappa$ and the geometrical parameters $r, l_{a}, l_{b}$. The diversity of parameters makes the solving process and the expressions of the formulas very complex. In general, nondimensionalizing the governing equations and the boundary conditions would benefit the solution and analysis of engineering problems. This process often yields important dimensionless parameters and reduces the dependency of the solution on a potentially large number of dimensional parameters.

Since the numerical techniques are heavily based on the properties of the related Chebyshev and Jacobi polynomials, it would be useful to set the crack in the normalized coordinate interval $[-1,1]$. Hence, we define the dimension-independent variables as follows:

$$
\left\{\begin{array}{c}
\left(x^{*}, y^{*}, r^{*}, l_{a}^{*}, l_{b}^{*}\right)=\frac{\left(x, y, r, l_{a}, l_{b}\right)}{r}, \quad t^{*}=\frac{a t}{r^{2}} \\
T^{*}=\frac{T-T_{0}}{T_{\infty}-T_{0}}, \\
\left(u^{*}, v^{*}\right)=\frac{(u, v)}{\alpha r\left(T_{\infty}-T_{0}\right)}, \quad\left(\sigma_{x}^{*}, \sigma_{x}^{*}, \tau_{x y}^{*}\right)=\frac{\left(\sigma_{x}, \sigma_{x}, \tau_{x y}\right)}{\mu \alpha\left(T_{\infty}-T_{0}\right)}
\end{array}\right.
$$

The governing equation and the boundary conditions of the temperature fields have the following dimensionless forms:

$$
\nabla^{2} T_{i}^{*}=\frac{\partial T_{i}^{*}}{\partial t^{*}}+\tau_{1} \frac{\partial^{2} T_{i}^{*}}{\partial t^{* 2}}
$$




$$
\begin{aligned}
& \left\{\begin{array}{lr}
\frac{\partial T_{1}^{*}}{\partial y^{*}}=B i_{1}\left(T_{1}^{*}-1\right) & \left|x^{*}\right|<\infty, y^{*}=-l_{a}^{*} \quad(a) \\
\frac{\partial T_{2}^{*}}{\partial y^{*}}=-B i_{2} T_{2}^{*} & \left|x^{*}\right|<\infty, y^{*}=l_{b}^{*} \quad(b) \\
\frac{\partial T_{1}^{*}}{\partial y^{*}}=\frac{\partial T_{2}^{*}}{\partial y^{*}} & \left|x^{*}\right|>1, y^{*}=0 \quad(c) \\
T_{1}^{*}=T_{2}^{*} & \left|x^{*}\right|>1, y^{*}=0 \quad(d) \\
\frac{\partial T_{1}^{*}}{\partial y^{*}}=\frac{\partial T_{2}^{*}}{\partial y^{*}}=B i_{3}\left(T_{2}^{*}-T_{1}^{*}\right) & \left|x^{*}\right| \leqslant 1, y^{*}=0
\end{array}\right. \\
& \left\{\begin{array}{lll}
T_{i}^{*}=0 \quad\left|x^{*}\right|<\infty,-l_{a}^{*}<y^{*}<l_{b}^{*}, t^{*}=0 \quad(a) \\
\frac{\partial T_{i}^{*}}{\partial t}=0 \quad\left|x^{*}\right|<\infty,-l_{a}^{*}<y^{*}<l_{b}^{*}, t^{*}=0 \quad(b)
\end{array}\right.
\end{aligned}
$$

where the gradient operator $\nabla$ is related to the dimensionless coordinates $x^{*}$ and $y^{*} . \tau_{1}$ is the normalized thermal relaxation time which we named the "Non-Fourier factor" (NFF) [24,32] and is defined as:

$$
\tau_{1}=\frac{a \tau_{0}}{r^{2}}
$$

The coefficient $B i$ is defined as

$$
B i_{i}=\frac{h_{i} r}{k},(i=1,2,3)
$$

and named "Effective Biot's number". It is worthy to note that in this work $B i_{i}(i=1,2,3)$ differs slightly from the conventional Biot's number, which should be defined as Biot $t_{i}=h_{i}\left(l_{a}+l_{b}\right) / k(i=1,2,3)$. In this study, the values of $l_{a}$ and $l_{b}$ are preset to be $r$ and $4 r$ therefore the relations between $B i_{i}$ and $B i o t_{i}$ is $B i_{i}=B i o t_{i} / 5$. In the following analysis, the superscript " $*$ " will be omitted for simplicity.

In heat conduction problems, the Laplace transform is generally applied to the time variable $t$. The Laplace transform is defined as:

$$
\bar{g}(x, y)=\int_{0}^{\infty} g(x, y, t) e^{-p t} \mathrm{~d} t
$$

By definition, $g(x, y, t)$ can be expressed in term of $\bar{g}(x, y)$

$$
g(x, y, t)=\frac{1}{2 \pi \mathrm{i}} \int_{\gamma-\mathrm{i} \infty}^{\gamma+\mathrm{i} \infty} \bar{g}(x, y) e^{p t} \mathrm{~d} p
$$

where the parameter " $\mathrm{i}$ " is the imaginary unit. It should not be confounded with the italic letter " $i$ ", which is used as a subscript order. $\gamma$ is a real constant that exceeds the real part of all the singularities of $\bar{g}(x, y)$. The functions $g(x, y, t)$ and $\bar{g}(x, y)$ are called a Laplace transform pair [33]. By substituting the Laplace transform (17) to Eqs. (11) and (12), and taking use of the initial conditions (13), we have the governing equations and boundary conditions in Laplace domain:

$\nabla^{2} \bar{T}_{i}=\left(p+\tau_{1} p^{2}\right) \bar{T}_{i} \quad i=1,2$

$$
\begin{array}{lll}
\frac{\partial \bar{T}_{1}}{\partial y}=B i_{1}\left(\bar{T}_{1}-\frac{1}{p}\right) & |x|<\infty, y=-l_{a} & (a) \\
\frac{\partial \bar{T}_{2}}{\partial y}=-B i_{2} \bar{T}_{2} & |x|<\infty, y=l_{b} & (b) \\
\frac{\partial \bar{T}_{1}}{\partial y}=\frac{\partial \bar{T}_{2}}{\partial y} & |x|>1, y=0 & (c) \\
\bar{T}_{1}=\bar{T}_{2} & |x|>1, y=0 & (d) \\
\frac{\partial \bar{T}_{1}}{\partial y}=\frac{\partial \bar{T}_{2}}{\partial y}=B i_{3}\left(\bar{T}_{2}-\bar{T}_{1}\right) & |x| \leqslant 1, y=0 & (e) \\
\left\{\begin{array}{lll}
\frac{\partial \bar{T}_{1}}{\partial y}=B i_{1}\left(\bar{T}_{1}-\frac{1}{p}\right) & |x|<\infty, y=-l_{a} & (a) \\
\frac{\partial \bar{T}_{2}}{\partial y}=-B i_{2} \bar{T}_{2} & |x|<\infty, y=l_{b} & (b) \\
\frac{\partial \bar{T}_{1}}{\partial y}=\frac{\partial \bar{T}_{2}}{\partial y} & |x|>1, y=0 & (c) \\
\bar{T}_{1}=\bar{T}_{2} & |x| \leqslant 1, y=0 & (e) \\
\frac{\partial \bar{T}_{1}}{\partial y}=\frac{\partial \bar{T}_{2}}{\partial y}=B i_{3}\left(\bar{T}_{2}-\bar{T}_{1}\right) &
\end{array}\right.
\end{array}
$$

By taking use of the Fourier transform, the general solutions of the temperature fields can be written as:

$$
\left\{\begin{array}{cc}
\bar{T}_{1}=\frac{1}{2 \pi} \int_{-\infty}^{\infty}\left(C_{T, 1} e^{-m y}+C_{T, 2} e^{m y}\right) e^{i \xi x} d \xi & |x|<\infty,-l_{a}<y<0 \\
\bar{T}_{2}=\frac{1}{2 \pi} \int_{-\infty}^{\infty}\left(C_{T, 3} e^{-m y}+C_{T, 4} e^{m y}\right) e^{i \xi x} d \xi & |x|<\infty, 0<y<l_{b}
\end{array}\right.
$$


where $m=\left(\xi^{2}+p+\tau_{1} p^{2}\right)^{1 / 2}, C_{T, i}(i=1,2,3,4)$ are unknown functions and can be obtained by the boundary conditions. Introduce the density function as:

$$
\phi(x)=\frac{\partial \bar{T}_{2}}{\partial x}-\frac{\partial \bar{T}_{1}}{\partial x}, \quad|x|<\infty, y=0
$$

It is clear that from the symmetry of the model that

$$
\int_{-1}^{1} \phi(x) \mathrm{d} x=0
$$

and at the extension line of the crack, the density function satisfies:

$$
\phi(x)=0, \quad|x|>1
$$

By substituting (21) into the boundary conditions (20) (a) - (c) and the density function (22), $C_{T, i}$ can be obtained and the temperature fields in Laplace space can be expressed as:.

$$
\left\{\begin{array}{c}
\bar{T}_{1}=\frac{1}{2 \pi} \int_{0}^{\infty} \bar{T}_{1, \phi} \cos (\xi x) \int_{-1}^{1} \phi \sin (\xi x) d x \mathrm{~d} \xi+\bar{T}_{\delta} \\
\bar{T}_{2}=\frac{1}{2 \pi} \int_{0}^{\infty} \bar{T}_{2, \phi} \cos (\xi x) \int_{-1}^{1} \phi \sin (\xi x) d x \mathrm{~d} \xi+\bar{T}_{\delta}
\end{array}\right.
$$

where

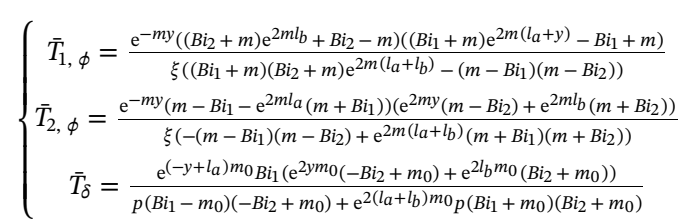

where $m_{0}=\left(p+\tau_{1} p^{2}\right)^{1 / 2}$. Noting that $\phi$ is the only undetermined function in (25), therefore, the boundary condition $(e)$ in (20) is sufficient to determinate the unknown. Substituting (25) into (20) (e), we obtain the integral equation in which the only unknown is $\phi(\eta)$.

$$
\int_{-1}^{1} \phi(\eta)\left(\frac{1}{\eta-x}+k(x, \eta)\right) \mathrm{d} \eta=g_{1}(x)
$$

where $k(x, \eta)$ is the Fredholm kernel and given by

$$
\begin{gathered}
k(x, \eta)= \\
\int_{0}^{\infty}\left(-\frac{2}{\xi} \frac{m\left(B i_{1} \cosh \left(m l_{a}\right)+m \sinh \left(m l_{a}\right)\right)\left(B i_{2} \cosh \left(m l_{b}\right)+m \sinh \left(m l_{b}\right)\right)}{\left(i_{2}+m^{2}\right) \sinh \left(m\left(l_{a}+l_{b}\right)\right)+\left(B i_{1}+B i_{2}\right) m \cosh \left(m\left(l_{a}+l_{b}\right)\right)}\right. \\
\left.-\frac{2}{\xi} B i_{3}+1\right) \cdot \sin (\xi(x-\eta)) \mathrm{d} \xi
\end{gathered}
$$

and

$$
g_{1}(x)=\frac{2 \pi B i_{1} m_{0}\left(B i_{2} \cosh \left(m_{0} l_{b}\right)+m_{0} \sinh \left(m_{0} l_{b}\right)\right)}{p\left(B i_{1} B i_{2}+m_{0}^{2}\right) \sinh \left(m_{0}\left(l_{a}+l_{b}\right)\right)+\left(B i_{1}+B i_{2}\right) m_{0} p \cosh \left(m_{0}\left(l_{a}+l_{b}\right)\right)}
$$

Eq. (27) is the singular integral equation of the first kind which arises from the formulation of the mixed boundary value conditions. According to the singular properties of the crack tip, the solution of (27) can be rewritten as

$$
\phi(x)=\frac{\Phi(x)}{\sqrt{1-x^{2}}}
$$

An effective numerical solution of (27) can be obtained by using a quadrature formula of the Gaussian type with appropriately selected orthogonal polynomial and values of $x_{j}(j=1,2, \ldots, n-1)$. Thereby Eq. (27) and (23) can be reduced to the following system of linear algebraic equations of the unknown function $\Phi\left(\eta_{i}\right)$, written as

$$
\left\{\begin{array}{c}
\frac{\pi}{n} \sum_{i=1}^{n} \Phi\left(\eta_{i}\right)\left(\frac{1}{\eta_{i}-x_{j}}+k\left(x_{j}, \eta_{i}\right)\right)=g\left(x_{j}\right), \quad j=1, \cdots, n-1 \\
\sum_{i=1}^{n} \Phi\left(\eta_{i}\right)=0
\end{array}\right.
$$

Once the function $\Phi\left(\eta_{i}\right)$ is obtained, the temperature fields in Laplace space can be obtained by using the following numerical integration:

$$
\int_{-1}^{1} \phi \sin (\xi x) d x \cong \frac{\pi}{n} \sum_{i=1}^{n} \Phi\left(\eta_{i}\right) \sin (\xi x)
$$

The temperature fields in time domain can be given by applying the Laplace inverse transform developed by Miller and Guy [34]. 


\subsection{Displacement fields}

Similarly, the dimensionless governing equations and boundary conditions of the displacement fields have the following form:

$$
\begin{aligned}
& \begin{cases}(1+\kappa) \frac{\partial^{2} u^{*}}{\partial x^{* 2}}+(\kappa-1) \frac{\partial^{2} u^{*}}{\partial y^{* 2}}+2 \frac{\partial^{2} v^{*}}{\partial x^{*} \partial y^{*}}=4 \frac{\partial T^{*}}{\partial x^{*}} \\
(\kappa-1) \frac{\partial^{2} v^{*}}{\partial x^{* 2}}+(1+\kappa) \frac{\partial^{2} v^{*}}{\partial y^{* 2}}+2 \frac{\partial^{2} u^{*}}{\partial x^{*} \partial y^{*}}=4 \frac{\partial T^{*}}{\partial y^{*}}\end{cases} \\
& \left\{\begin{array}{llll}
\sigma_{y 1}^{*}=0 & \left|x^{*}\right|<\infty, \quad y^{*}=-l_{a}^{*} \quad(a) \\
\tau_{x y 1}^{*}=0 & \left|x^{*}\right|<\infty, \quad y^{*}=-l_{a}^{*} \quad(b) \\
\sigma_{y 2}^{*}=0 & \left|x^{*}\right|<\infty, \quad y^{*}=l_{b}^{*} \quad(c) \\
\tau_{x y 2}^{*}=0 & \left|x^{*}\right|<\infty, \quad y^{*}=l_{b}^{*} \quad(d) \\
\sigma_{y 1}^{*}=\sigma_{y 2}^{*} & \left|x^{*}\right|>1, \quad y^{*}=0 \quad(e) \\
\tau_{x y 1}^{*}=\tau_{x y 2}^{*} & \left|x^{*}\right|>1, \quad y^{*}=0 \quad(f) \\
\sigma_{y 1}^{*}=\sigma_{y 2}^{*}=0 & \left|x^{*}\right| \leqslant 1, \quad y^{*}=0 \quad(g) \\
\tau_{x y 1}^{*}=\tau_{x y 2}^{*}=0 & \left|x^{*}\right| \leqslant 1, \quad y^{*}=0 \quad(h) \\
u_{1}^{*}=u_{2}^{*} & \left|x^{*}\right|>1, \quad y^{*}=0 \quad(i) \\
v_{1}^{*}=v_{2}^{*} & \left|x^{*}\right|>1, \quad y^{*}=0 \quad(j)
\end{array}\right. \\
& \left\{\begin{array}{llll}
u_{i}^{*}=v_{i}^{*}=0 & \left|x^{*}\right|<\infty,-l_{a}^{*}<y<l_{b}^{*}, \quad t^{*}=0 \quad(a) \\
\sigma_{x i}^{*}=\sigma_{y i}^{*}=\tau_{x y i}^{*}=0 & \left|x^{*}\right|<\infty,-l_{a}^{*}<y<l_{b}^{*}, \quad t^{*}=0 \quad(b)
\end{array}\right.
\end{aligned}
$$

In the following analysis, the superscript “*” will be omitted for simplicity. Applying the Laplace transform to (33) and (34),

$$
\begin{aligned}
& \left\{\begin{array}{ll}
(1+\kappa) \frac{\partial^{2} \bar{u}_{i}}{\partial x^{2}}+(\kappa-1) \frac{\partial^{2} \bar{u}_{i}}{\partial y^{2}}+2 \frac{\partial^{2} \bar{v}_{i}}{\partial x \partial y}=4 \frac{\partial \bar{T}_{i}}{\partial x} \\
(\kappa-1) \frac{\partial^{2} \bar{v}_{i}}{\partial x^{2}}+2 \frac{\partial^{2} \bar{u}_{i}}{\partial x \partial y}+(1+\kappa) \frac{\partial^{2} \bar{v}_{i}}{\partial y^{2}}=4 \frac{\partial \bar{T}_{i}}{\partial y}
\end{array} \quad i=1,2\right. \\
& \left\{\begin{array}{llll}
\bar{\sigma}_{y 1}=0 & |x|<\infty, \quad y=-l_{a} & (a) \\
\bar{\tau}_{x y 1}=0 & |x|<\infty, \quad y=-l_{a} & (b) \\
\bar{\sigma}_{y 2}=0 & |x|<\infty, \quad y=l_{b} & (c) \\
\bar{\tau}_{x y 2}=0 & |x|<\infty, & y=l_{b} & (d) \\
\bar{\sigma}_{y 1}=\bar{\sigma}_{y 2} & |x|>1, & y=0 & (e) \\
\bar{\tau}_{x y 1}=\bar{\tau}_{x y 2} & |x|>1, & y=0 & (f) \\
\bar{\sigma}_{y 1}=\bar{\sigma}_{y 2}=0 & |x| \leqslant 1, & y=0 & (g) \\
\bar{\tau}_{x y 1}=\bar{\tau}_{x y 2}=0 & |x| \leqslant 1, & y=0 & (h) \\
\bar{u}_{1}=\bar{u}_{2} & |x|>1, & y=0 & (i) \\
\bar{v}_{1}=\bar{v}_{2} & |x|>1, & y=0 & (j)
\end{array}\right.
\end{aligned}
$$

The above PDEs have the following characteristic polynomial obtained by setting their general solutions of the form $e^{\lambda y}$ :

$$
\lambda^{4}-2 \lambda^{2} \xi^{2}+\xi^{4}=0
$$

The roots of the polynomial equation (38) are given by

$$
\lambda_{1}=-\xi, \lambda_{2}=-\xi, \lambda_{3}=\xi, \lambda_{4}=\xi
$$

The displacement fields in Laplace domain satisfying the boundary condition and regularity condition can be expressed as

$$
\left\{\begin{array}{l}
\bar{u}_{1}(y)=\frac{1}{2 \pi} \int_{-\infty}^{\infty}\left[\left(C_{1}^{H}+C_{2}^{H} y\right) e^{-|\xi| y}+\left(C_{3}^{H}+C_{4}^{H} y\right) e^{|\xi| y}+\bar{u}_{1, \phi}^{P}+\bar{u}_{1, \delta}^{P}\right] e^{i \xi x} \boldsymbol{d} \xi \\
\bar{u}_{2}(y)=\frac{1}{2 \pi} \int_{-\infty}^{\infty}\left[\left(C_{5}^{H}+C_{6}^{H} y\right) e^{-|\xi| y}+\left(C_{7}^{H}+C_{8}^{H} y\right) e^{|\xi| y}+\bar{u}_{2, \phi}^{P}+\bar{u}_{2, \delta}^{P}\right] e^{i \xi x} \boldsymbol{d} \xi \\
\bar{v}_{1}(y)=\frac{1}{2 \pi} \int_{-\infty}^{\infty}\left[\left(B_{1}^{H}+B_{2}^{H} y\right) e^{-|\xi| y}+\left(B_{3}^{H}+B_{4}^{H} y\right) e^{|\xi| y}+\bar{u}_{3, \phi}^{P}+\bar{u}_{3, \delta}^{P}\right] e^{i \xi x} \boldsymbol{d} \xi \\
\bar{v}_{2}(y)=\frac{1}{2 \pi} \int_{-\infty}^{\infty}\left[\left(B_{5}^{H}+B_{6}^{H} y\right) e^{-|\xi| y}+\left(B_{7}^{H}+B_{8}^{H} y\right) e^{|\xi| y}+\bar{u}_{4, \phi}^{P}+\bar{u}_{4, \delta}^{P}\right] e^{i \xi x} \boldsymbol{d} \xi
\end{array}\right.
$$

where $B_{i}^{H}$ and $C_{i}^{H}(i=1, \cdots, 8)$ are unknown functions to be determined. The functions $B_{i}^{H}$ and $C_{i}^{H}(i=1, \cdots, 8)$ are inter-dependent. Their relationships, obtained by substituting Eq. (40) into either one of Eq. (36), are given in (A.1). The expressions of $\bar{u}_{i, \phi}^{P}, \bar{u}_{i, \delta}^{P}, \bar{v}_{i, \phi}^{P}, \bar{v}_{i, \delta}^{P}(i=1,2)$ are given in (A.2) - (A.9).

To solve the unknown functions $C_{i}^{H}(i=1, \cdots, 8)$, the boundary conditions $(a)-(f)$ in (37) can be used. This allows providing six 
equations. The other two equations are found by considering the mixed boundary conditions $(g)$ and $(h)$ in (37) which would give a pair of dual integral equations. Now we define two dimensionless dislocation functions $f_{i}(i=1,2)$ along the crack lips $y=0$ as follows:

$$
\begin{cases}f_{1}(x)=\frac{\partial \bar{u}_{2}}{\partial x}-\frac{\partial \bar{u}_{1}}{\partial x} \quad y=0 \\ f_{2}(x)=\frac{\partial \bar{v}_{2}}{\partial x}-\frac{\partial \bar{v}_{1}}{\partial x} \quad y=0\end{cases}
$$

From the conditions $(i)$ and $(j)$ of $(37)$, we know that $f_{i}(i=1,2)$ also satisfy the single-valued conditions:

$$
\int_{-1}^{1} f_{i}(x) d x=0 \quad(i=1,2)
$$

and

$$
f_{i}(x)=0 \quad(i=1,2) \quad|x| \geqslant 1
$$

Physically speaking, Eq. (43) means that the displacements are single-valued for the non-cracked portion along $y=0$.

$C_{i}^{H}(i=1, \cdots, 8)$ can expressed in terms of $f_{i}(i=1,2)$ by applying the definition of the dislocation function $f_{i}(i=1,2)$ in $(41)$ and the boundary conditions $(a)-(f)$ in (37):

$$
C_{i}^{H}=\sum_{j=1}^{8} \mathbf{A}_{i, j} \cdot \gamma_{j}+\frac{1}{\mathrm{i} \xi} \sum_{j=1}^{2} \mathbf{A}_{i, j} \int_{-1}^{1} f_{j} e^{-i \xi x} \mathbf{d} x \quad i=1,2, \cdots, 8
$$

where $\mathbf{A}$ is a square matrix and $\gamma$ is a column vector and are given in (A.10) - (A.12). The subscript $i$ and $j$ of $\mathbf{A}$ denote the $i$ th row and $j$ th column of A. By applying the boundary conditions $(g-h)$ in $(37)$, it can be shown that $f_{i}(x)(i=1,2)$ satisfy the following singular integral equations(SIEs):

$$
\sum_{j=1}^{2} \int_{-1}^{1} \bar{f}_{j}(\eta)\left(\int_{0}^{\infty} K_{i, j}(\xi, x, \eta) \mathrm{d} \xi+\frac{\delta_{i, j}}{\eta-x}\right) \mathrm{d} \eta=\int_{0}^{\infty} K_{i, 3}(\xi, x) \phi_{0} \mathrm{~d} \xi, \quad i=1,2
$$

where $K_{i j}(i=1,2 ; j=1,2,3)$ are given in - and $\phi_{0}$ is given by:

$$
\phi_{0}=\sum_{i=1}^{n} \sin \left(\xi \cos \left(\frac{\pi\left(2 \eta_{i}-1\right)}{2 n}\right)\right) \Phi\left(\eta_{i}\right)
$$

According to the singularity of the crack tips, the solution of the SIEs can be rewritten as

$$
f_{i}(x)=\frac{F_{i}(x)}{\sqrt{1-x^{2}}} \quad(i=1,2)
$$

where $F_{i}(i=1,2)$ satisfies the Hölder condition on the closed interval of $[-1,1]$ and $F_{i}(-1) \neq 0, F_{i}(1) \neq 0$. An effective numerical solution can be obtained by using a quadrature formula of the Gaussian type with appropriately selected orthogonal polynomial and values of $x_{j}(j=1, \cdots, n-1)$. Thereby the SIEs (45) can be reduced to the following system of linear algebraic equations of the unknown function, written as

$$
\left\{\begin{aligned}
\sum_{l=1}^{2}\left(\sum_{i=1}^{n} A_{i}\left(\int_{0}^{\infty} K_{k, l}\left(\xi, x_{j}, \eta_{i}\right) \mathrm{d} \xi+\frac{\delta_{i, j}}{\eta_{i}-x_{j}}\right) F_{l}\left(\eta_{i}\right)\right) & =\int_{0}^{\infty} K_{k, 3}(\xi, x) \phi_{0} \mathrm{~d} \xi, \quad k=1,2 \\
\sum_{i=1}^{n} A_{i} F_{l}\left(\eta_{i}\right)=0, \quad l & =1,2
\end{aligned}\right.
$$

where $K_{k, l}(k=1,2 ; l=1,2,3)$ are given in (A.14) - (A.19). The discrete points $\eta_{i}(i=1, \cdots, n)$ correspond to the zeros of the orthogonal polynomials which related to the particular Gaussian quadrature. The corresponding Gauss point are:

$$
\left\{\begin{array}{lc}
\eta_{i}=\cos \left(\frac{i-1}{2 n} \pi\right), & i=1, \cdots, n \\
x_{j}=\cos \left(\frac{j}{n} \pi\right) & j=1, \cdots, n-1 \\
A_{i}=\frac{\pi}{2(n-1)} & i=1, n \\
A_{i}=\frac{\pi}{n-1} & i=2, \cdots, n-1
\end{array}\right.
$$

In this paper, the number of Gauss point is set to be 15 .

\subsection{Stress intensity factors}

The dimensionless stress intensity factors (SIFs) at the right-side crack tip in Laplace space are defined as: 


$$
\left\{\begin{array}{l}
\bar{K}_{1}=\lim _{x \rightarrow 1} \sqrt{2 \pi(x-1)} \bar{\sigma}_{y 1}(x, 0) \\
\bar{K}_{2}=\lim _{x \rightarrow 1} \sqrt{2 \pi(x-1)} \bar{\tau}_{x y 1}(x, 0)
\end{array}\right.
$$

The stress components at the crack ligaments can be rewritten as

$$
\left\{\begin{array}{c}
\frac{\pi}{2}(\kappa+1) \bar{\sigma}_{y 1}(x, 0)=\int_{-1}^{1} \frac{1}{\eta-x} f_{2}(\eta) \mathrm{d} \eta \\
+\sum_{j=1}^{2} \int_{-1}^{1} f_{j}(\eta)\left(\int_{0}^{\infty} K_{2, j}(\xi, x, \eta) \mathrm{d} \xi\right) \mathrm{d} \eta-\int_{0}^{\infty} K_{2,3}(\xi, x) \phi_{0} \mathrm{~d} \xi \\
\frac{\pi}{2}(\kappa+1) \bar{\tau}_{x y 1}(x, 0)=\int_{-1}^{1} \frac{1}{\eta-x} f_{1}(\eta) \mathrm{d} \eta \\
+\sum_{j=1}^{2} \int_{-1}^{1} f_{j}(\eta)\left(\int_{0}^{\infty} K_{1, j}(\xi, x, \eta) \mathrm{d} \xi\right) \mathrm{d} \eta-\int_{0}^{\infty} K_{1,3}(\xi, x) \phi_{0} \mathrm{~d} \xi
\end{array}\right.
$$

The stresses are expected to be singular at the crack tip. It is clear from the definition of the stress intensity factors that no terms in (51) is more singular than the square root singularity. Regarding the behavior of Cauchy integral [35]:

$$
\frac{1}{\pi} \int_{-1}^{1} \frac{f_{i}(\eta)}{\eta-x} \mathrm{~d} \eta=\frac{F_{i}(-1) \mathrm{e}^{\frac{\mathrm{i} \pi}{2}}}{\sqrt{2} \sqrt{1+x}}-\frac{F_{i}(1) \mathrm{e}^{-\frac{\mathrm{i} \pi}{2}}}{\sqrt{2} \sqrt{1-x}}+F_{0}(x), \quad i=1,2 ;|x| \geqslant 1
$$

where $F_{0}(x)$ is a bounded function. By omitting the less singular terms, we deduce the dimensionless stress intensity factors as follows:

$$
\left\{\begin{array}{l}
\bar{K}_{1}(1)=-\frac{\sqrt{\pi}}{\kappa+1} F_{2}(1) \\
\bar{K}_{2}(1)=-\frac{\sqrt{\pi}}{\kappa+1} F_{1}(1)
\end{array}\right.
$$

The stress intensity factors in the time domain can be obtained from (53) by using the numerical Laplace transform developed by Miller and Guy [34].

\section{Numerical results}

In this paper, the dimensionless geometrical parameters $l_{a}$ and $l_{b}$ are preset to be 1 and 4 , respectively; the Poisson's ratio $v$ is assumed to be a constant because the effect of its variation on the SIFs was shown to be negligible [36,37]. Here we choose $\kappa=2$, which corresponding to $\nu=0.33$ in plane stress and $\nu=0.25$ in plane strain. Therefore, the parameters that influence the distribution of the temperature field and the magnitude of the SIFs include the three effective Biot's number $B i_{i}(i=1,2,3)$ and the nonFourier Factor $\tau_{1}$. When $B i_{1}=\infty$ and $B i_{2}=0$, the model is similar with the study of Hu and Chen [38]. The numerical results in this paper corresponding well with the results given in previous literatures.

The coupling of the non-Fourier effect and the convection boundary conditions provides complex temperature and stress fields near the crack. In the following we will study the effect of these four factors in detail.

\subsection{Temperature fields}

The temperature at the mid-points of the crack surface(TM) versus dimensionless time is shown in Fig. 2 for $B i_{1}=B i_{2}=\infty$,

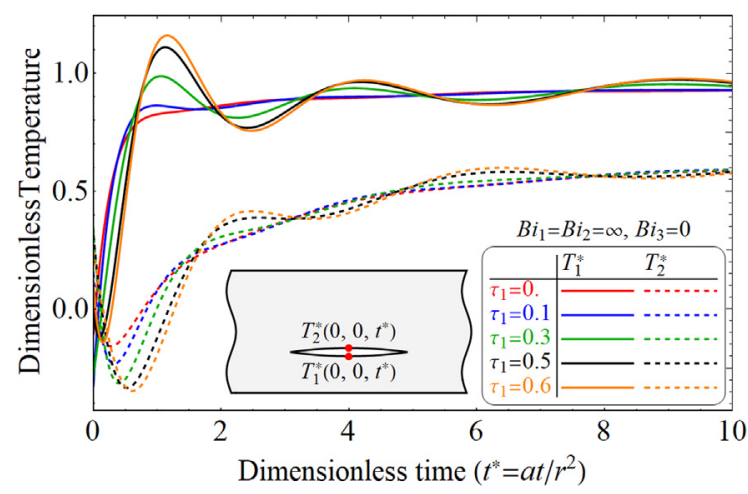

Fig. 2. Effect of the non-Fourier factor $\tau_{1}$ on the normalized temperatures at the mid-points of the crack surfaces $\left(T_{1}\left(0,0^{-}, t\right)-T_{0}\right) /\left(T_{\infty}-T_{0}\right)$ and $\left(T_{2}\left(0,0^{+}, t\right)-T_{0}\right) /\left(T_{\infty}-T_{0}\right)$ when $B i_{1}=B i_{2}=\infty$ and $B i_{3}=0$. 


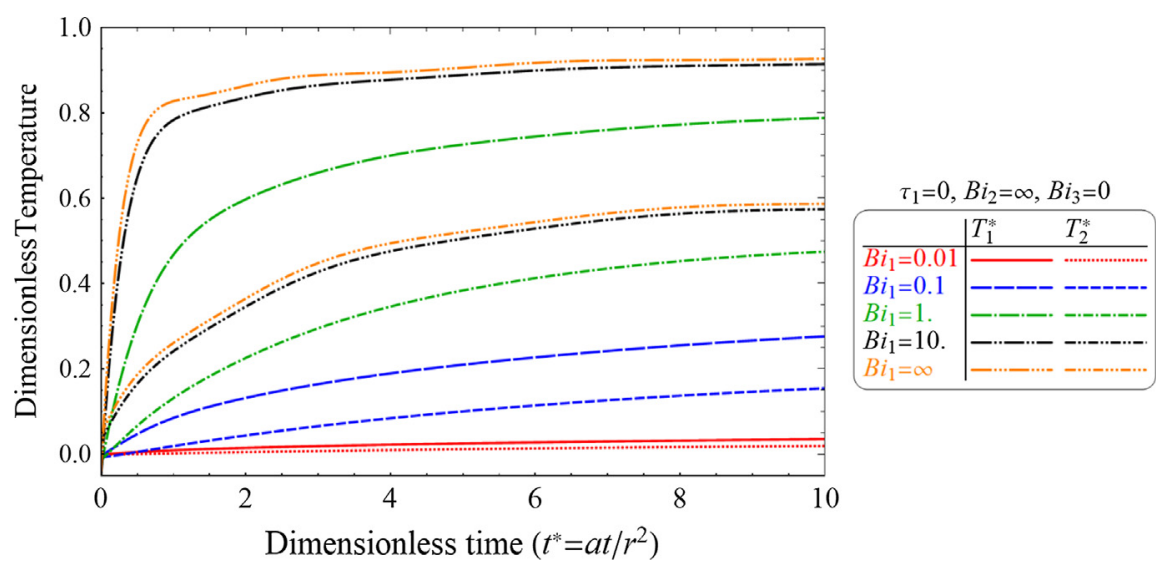

Fig. 3. Effect of $B i_{1}$ on the normalized temperatures at the mid-points of the crack surfaces $\left(T_{1}\left(0,0^{-}, t\right)-T_{0}\right) /\left(T_{\infty}-T_{0}\right)$ and $\left(T_{2}\left(0,0^{+}, t\right)-T_{0}\right) /$ $\left(T_{\infty}-T_{0}\right)$ when $\tau_{1}=0, B i_{2}=\infty$ and $B i_{3}=0$.

$B i_{3}=0$. The solid red curve and the long-dashed curve denote the temperature at the mid-point of the lower crack surface while the dotted red curve and the short-dashed curve denote the temperature at the mid-point of the upper crack surface, which is the same in Fig. 3, Fig. 5 and Fig. 7.

As $\tau_{1}$ increases from 0 to 0.6 , the magnitudes of the positive fluctuation on the lower face increase accordingly, while their steady state values convergent to a same value as time is long enough. Because of the presence of the attenuation term $\partial T / \partial t$ in the hyperbolic heat conduction Eq. (1), the oscillation amplitude and frequency of the curves decrease with the dimensionless time and converge to 0 as $t^{*}$ goes to infinity. The general results for the hyperbolic model can be reduced to the Fourier heat conduction results when either $\tau_{1}=0$, or $t^{*} \rightarrow \infty$, as shown in Fig. 2. The effect of $\tau_{1}$ were more thoroughly studied by Li [24] and Fu and Chen [10,20].

Fig. 3 shows the effect of $B i_{1}$ on $\mathbf{T M}$ when $\tau_{1}=0, B i_{2}=\infty$ and $B i_{3}=0$, respectively. Fig. 4 shows the maximum temperature difference between the mid-points of the crack surfaces (MTD) versus $B i_{1}$ for different $\tau_{1}$. When $B i_{1}$ equals to 0 , the lower surface is thermally insulated and the temperature of the plate remain unchanged after the thermal shock loading. Therefore, $B i_{1}=0.01$, a value that slightly larger than 0 , is chosen as the smallest $B i_{1}$ for plotting the curves. As the increasing of $B i_{1}$, the heat transfer performance of the lower surface increases, and therefore, the temperature field and the MTD increase too. It is also shown in Fig. 4 that the MTD is not sensitive of the non-Fourier effect for small $B i_{1}$ value, but becomes obviously sensitive and increase with $\tau_{1}$ when $B i_{1}$ greater than 0.2 .

Fig. 5 shows the effect of $B i_{2}$ on TM when $\tau_{1}=0, B i_{1}=\infty$ and $B i_{3}=0$. Fig. 6 illustrates the MTD versus $B i_{2}$ for different $\tau_{1}$. When $B i_{2}$ equals to 0 , the upper surface is thermally insulated. Therefor the heat accumulates over time in the plate and the temperature of the plate increases and can reach up to the dimensionless thermal shock temperature when the dimensionless time is long enough. The temperature field reaches a static equilibrium state and there is no heat flux in the plate. As the increasing of $B i_{2}$, the heat flow out of the upper surface is no longer zero. Therefore, the temperature field would reach a dynamic equilibrium state when the dimensionless time is long enough. In this case, the dimensionless temperature of the upper surface is less than 1 at the final state. It is shown from Fig. 6 that the MTD is not sensitive of $B i_{2}$ and slightly decreases with $B i_{2}$, but increases significantly with $\tau_{1}$.

Figs. 7 and 8 show the effect of $B i_{3}$ on TM when $B i_{1}=B i_{2}=\infty$ and $\tau_{1}=0$, respectively. Fig. 8 illustrates the MTD versus $B i_{3}$ for different values of $\tau_{1}$. When $B i_{3}$ equals to infinity, the crack surfaces have perfect thermal contact and the temperature field is the same as that obtained from the non-cracked plate model. Correspondingly, the temperature curves of the two mid-points coincide

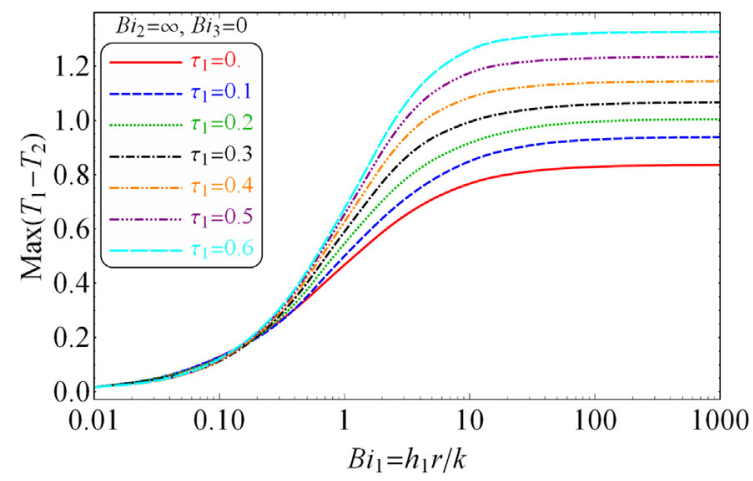

Fig. 4. Effect of $B i_{1}$ on the maximum temperature difference between the mid-points of the crack surfaces $\left(T_{1}\left(0,0^{-}, t\right)-T_{2}\left(0,0^{+}, t\right)\right) /\left(T_{\infty}-T_{0}\right)$ when $B i_{2}=\infty$ and $B i_{3}=0$. 


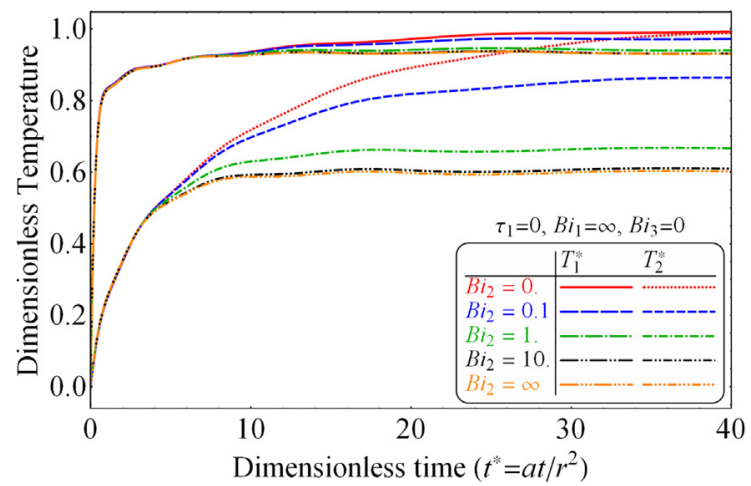

Fig. 5. Effect of $B i_{2}$ on the normalized temperatures at the mid-points of the crack surfaces $\left(T_{1}\left(0,0^{-}, t\right)-T_{0}\right) /\left(T_{\infty}-T_{0}\right)$ and $\left(T_{2}\left(0,0^{+}, t\right)-T_{0}\right) /$ $\left(T_{\infty}-T_{0}\right)$ when $\tau_{1}=0, B i_{1}=\infty$ and $B i_{3}=0$.

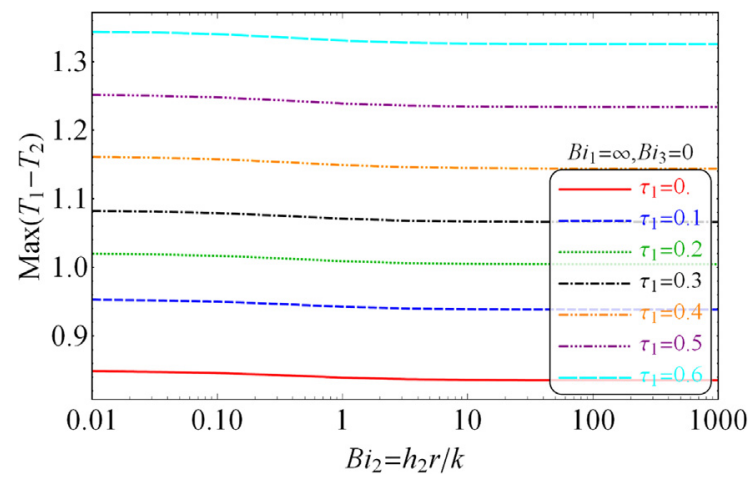

Fig. 6. Effect of $B i_{2}$ on the maximum temperature difference between the mid-points of the crack surfaces $\left(T_{1}\left(0,0^{-}, t\right)-T_{2}\left(0,0^{+}, t\right)\right) /\left(T_{\infty}-T_{0}\right)$ when $B i_{1}=\infty$ and $B i_{3}=0$.

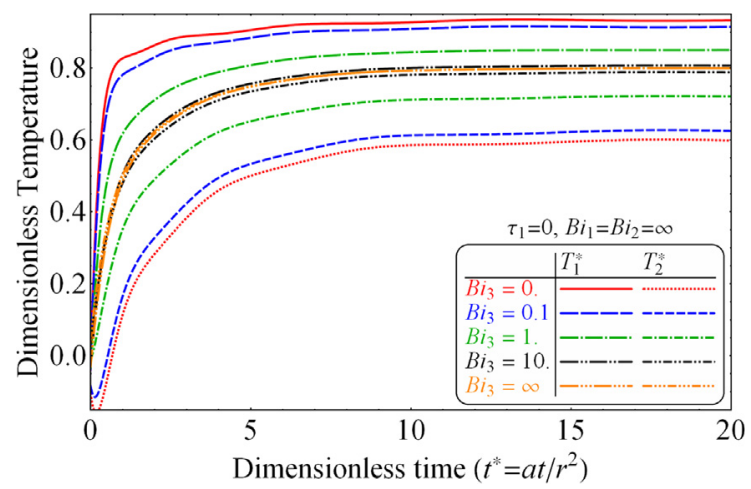

Fig. 7. Effect of $B i_{3}$ on the normalized temperatures at the mid-points of the crack surfaces $\left(T_{1}\left(0,0^{-}, t\right)-T_{0}\right) /\left(T_{\infty}-T_{0}\right)$ and $\left(T_{2}\left(0,0^{+}, t\right)-T_{0}\right) /$ $\left(T_{\infty}-T_{0}\right)$ when $\tau_{1}=0$ and $B i_{1}=B i_{2}=\infty$.

together (the orange curves in Fig. 7). The thermal insulation of crack gap increases with $B i_{3}$ and the temperature curves of the two mid-points get away from each other. Fig. 8 shows that the values of MTD are not sensitive of the non-Fourier effect for big $B i_{3}$ values, but increase evidently with $\tau_{1}$ when $B i_{3}<20$.

\subsection{Stress intensity factors}

The inertia effect of the stress wave and the coupled effect between the temperature and the stress are ignored in this study. Therefore, the temperature field is the only source which generates the thermal stress. Predictably, the SIFs have the similar properties to those of the temperature field. In the following we will analyze the properties of the SIFs by using the similar method to that described in the previous section.

Fig. 9 shows the effect of the non-Fourier factor, $\tau_{1}$, on the normalized SIFs $\left(K_{1}^{*}, K_{2}^{*}\right)=\left(K_{1}, K_{2}\right) /\left(\mu \alpha r^{1 / 2}\left(T_{\infty}-T_{0}\right)\right)$ when 


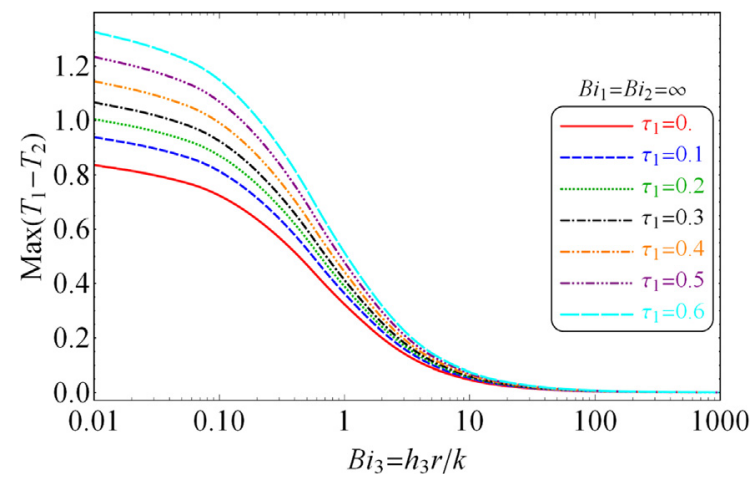

Fig. 8. Effect of $B i_{3}$ on the maximum temperature difference between the mid-points of the crack surfaces $\left(T_{1}\left(0,0^{-}, t\right)-T_{2}\left(0,0^{+}, t\right)\right) /\left(T_{\infty}-T_{0}\right)$ when $B i_{1}=B i_{2}=\infty$.

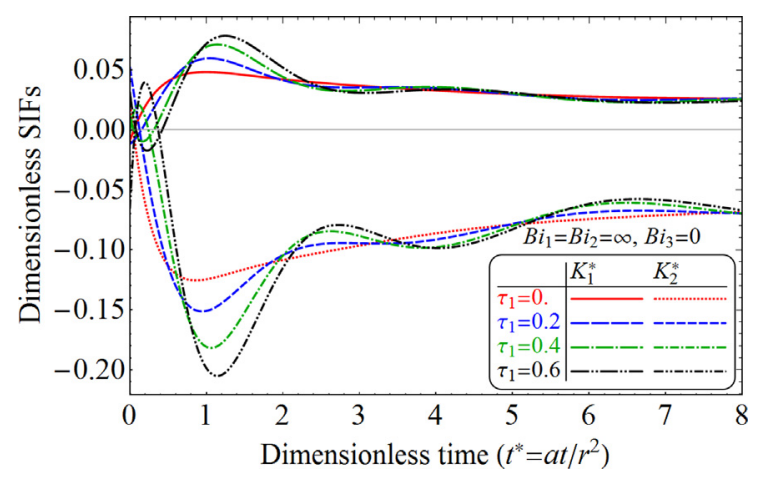

Fig. 9. Effect of the non-Fourier factor $\tau_{1}$ on the normalized stress intensity factors $\left(K_{1}^{*}, K_{2}^{*}\right)=\left(K_{1}, K_{2}\right) /\left(\mu \alpha r^{1 / 2}\left(T_{\infty}-T_{0}\right)\right)$ when $B i_{1}=B i_{2}=\infty$ and $B i_{3}=0$.

$B i_{1}=B i_{2}=\infty$ and $B i_{3}=0$. The solid red curve and the long-dashed curve denote $K_{1} *$ while the dotted red curve and the shortdashed curve denote $K_{2}{ }^{*}$. This graphical convention applies also to Figs. 10, 12 and 14. In Fig. 9, the red curve ( $\left.\tau_{1}=0\right)$ is obtained by neglecting the non-Fourier effect. As $\tau_{1}$ grows, the oscillation property appears in the evolution curves of the SIFs, similarly to that of the temperature field. The steady state values of the SIFs converge to a constant value when the time is long enough. From the tendency of these two groups of curves, we can conclude that the principal effect of the non-Fourier factor on the stress field is the oscillation of the later during the thermal shock process. It is clear that the stress oscillation occurs as the consequence of the temperature wave. More importantly, the maximum SIFs can be much higher than those obtained with the conventional Fourier heat conduction analysis.

The curves SIFs versus dimensionless time for different $B i_{1}$ and the curves maximum SIFs versus $B i_{1}$ for different $\tau_{1}$ are shown in Figs. 10 and 11, respectively. There is no temperature change in the plate and no stress concentration at the crack tips when the lower surface is completely heat insulated. Similarly, when $B i_{1}$ is small, the surface heat resistance is large and the variation of temperature field around the crack is small, which lead to small SIFs. The magnitude of SIFs increase as the effective Biot's number $B i_{1}$ increase and reach the peak value at $B i_{1}=\infty$. The orange curves in Fig. 10 correspond to the red curves in Fig. 9.

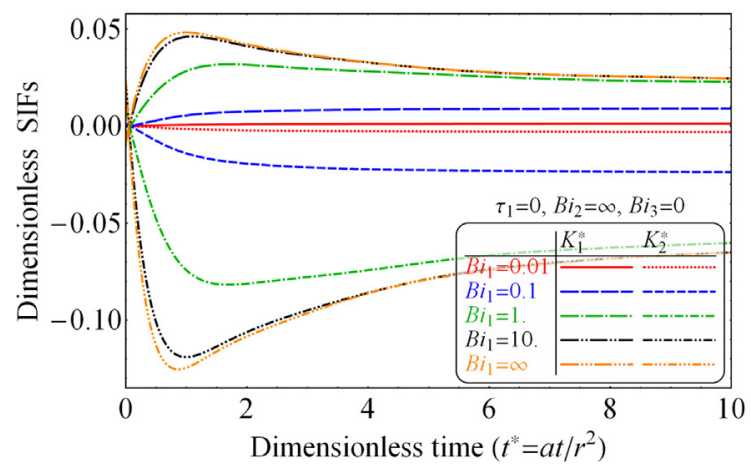

Fig. 10. Effect of $B i_{1}$ on the normalized stress intensity factors $\left(K_{1}^{*}, K_{2}^{*}\right)=\left(K_{1}, K_{2}\right) /\left(\mu \alpha r^{1 / 2}\left(T_{\infty}-T_{0}\right)\right)$ when $\tau_{1}=0, B i_{2}=\infty$ and $B i_{3}=0$. 


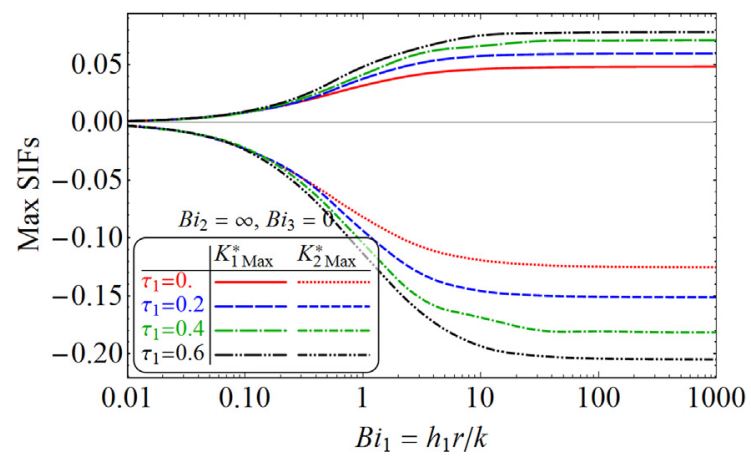

Fig. 11. Effect of $B i_{1}$ on the maximum stress intensity factors $\left(K_{1 \max }{ }^{*}, K_{2 \max }{ }^{*}\right)=\left(K_{1 \max }, K_{2 \max }\right) /\left(\mu \alpha r^{1 / 2}\left(T_{\infty}-T_{0}\right)\right)$ when $\tau_{1}=0, B i_{2}=\infty$ and $B i_{3}=0$.

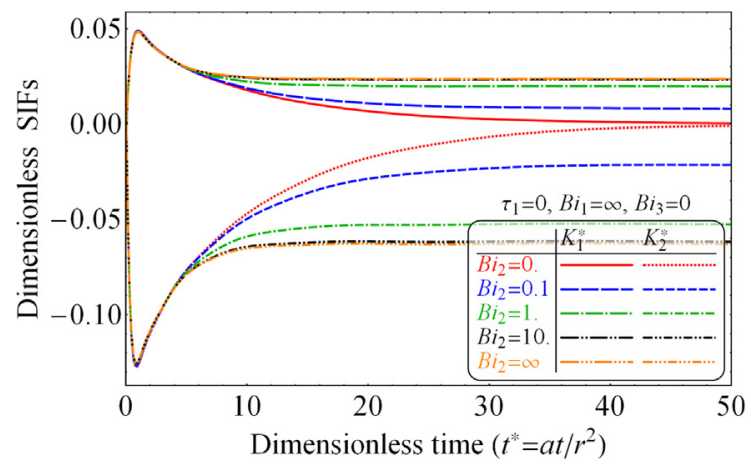

Fig. 12. Effect of $B i_{2}$ on the normalized stress intensity factors $\left(K_{1}^{*}, K_{2}^{*}\right)=\left(K_{1}, K_{2}\right) /\left(\mu \alpha r^{1 / 2}\left(T_{\infty}-T_{0}\right)\right)$ when $\tau_{1}=0, B i_{1}=\infty$ and $B i_{3}=0$.

The variation of SIFs versus dimensionless time for different $B i_{2}$ and the variation of peak-value of SIFs versus $B i_{2}$ for different $\tau_{1}$ are shown in Figs. 12 and 13, respectively. It is clear from these two figures that the magnitude of $B i_{2}$ doesn't change the peak value of the SIFs, whereas the final convergence value and the convergence speed of SIFs increase as $B i_{2}$ increases. Especially, the dimensionless time used to reach a stable state when $B i_{2}=0$ is almost 4 times higher than the corresponding time when $B i_{2}$ tends to infinity.

Figs. 14 and 15 exhibit the variation of SIFs versus dimensionless time for different $B i_{3}$ and the variation of peak-value of SIFs versus $B i_{3}$ for different $\tau_{1}$, respectively. When $B i_{3}$ equals to infinity, the temperature field is the same as that obtained from the noncracked plate model and the temperature gradient has no singularity at the crack tips. Therefore the SIFs are almost 0 . As the decrease of $B i_{3}$, the thermal insulation of the crack gap increases and then the SIFs increase. The absolute value of $K_{1}$ and $K_{2}$ increase at the beginning and decrease after they reach the top value. The peak values increase as the effective Biot's number $B i_{3}$ decreases. This trend is directly related to the dependence of the temperature field on effective Biot's number. As discussed above, the crack behaves as a thermal resistance: smaller effective Biot's numbers signify bigger thermal resistance. Therefore, the smaller the effective Biot's number is, the higher the temperature gradient is around the crack and consequently, the higher the SIFs will be.

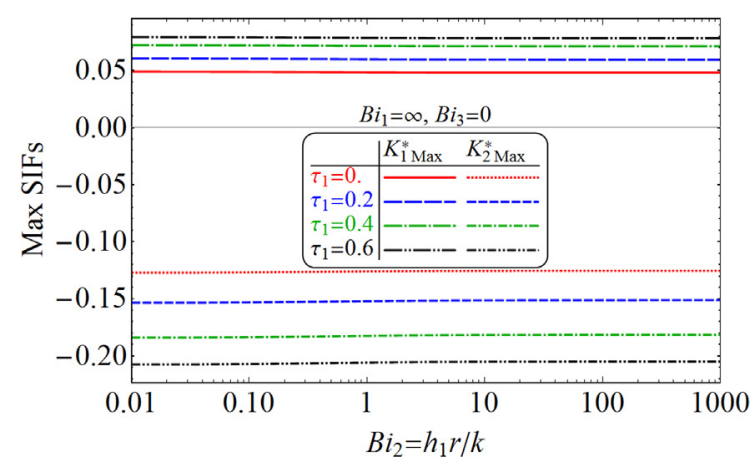

Fig. 13. Effect of $B i_{2}$ on the normalized stress intensity factors $\left(K_{1 \max }{ }^{*}, K_{2 \max }{ }^{*}\right)=\left(K_{1 \max }, K_{2 \max }\right) /\left(\mu \alpha r^{1 / 2}\left(T_{\infty}-T_{0}\right)\right)$ when $\tau_{1}=0, B i_{1}=\infty$ and $B i_{3}=0$. 


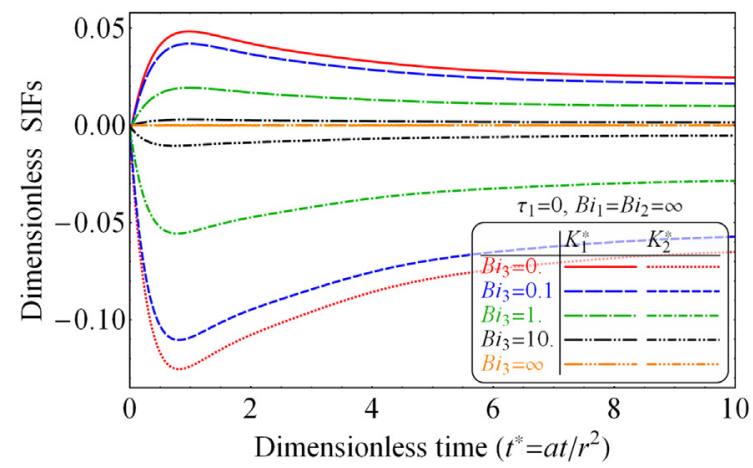

Fig. 14. Effect of $B i_{3}$ on the normalized stress intensity factors $\left(K_{1}^{*}, K_{2}^{*}\right)=\left(K_{1}, K_{2}\right) /\left(\mu \alpha r^{1 / 2}\left(T_{\infty}-T_{0}\right)\right)$ when $\tau_{1}=0$ and $B i_{1}=B i_{2}=\infty$.

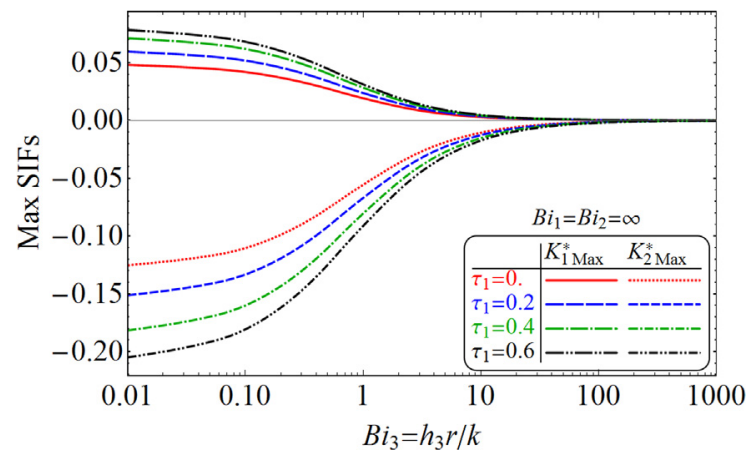

Fig. 15. Effect of $B i_{3}$ on the normalized stress intensity factors $\left(K_{1 \max }{ }^{*}, K_{2 \max }{ }^{*}\right)=\left(K_{1 \max }, K_{2 \max }\right) /\left(\mu \alpha r^{1 / 2}\left(T_{\infty}-T_{0}\right)\right)$ when $\tau_{1}=0$ and $B i_{1}=B i_{2}=$ $\infty$..ADDIN

\section{Conclusions}

The thermal shock problem of a cracked thermoelastic plate has been studied using the hyperbolic thermal conduction theory. Surface heat transfer properties are considered in all the boundaries during the analysis. The governing equation and the boundary conditions are written in dimensionless form. The Laplace transform and the Fourier transform are applied to solve the mixedboundary condition problem. The numerical integrations and the numerical Laplace transform are used to obtain the temperature and the stress intensity factors in the time domain.

The curves in Figs. 4, 6, 8, 11, 13 and 15 are almost parallel to each other, which means the non-Fourier effect and the heat transfer condition of the surfaces are uncoupled. On the one hand, larger $\tau_{1}$ always leads to larger maximum values of the temperature difference between the two crack surfaces and therefore to the larger values of the SIFs for a given group of boundary conditions. On the other hand, the influence of Biot's numbers on the temperature field and on the SIFs is quite similar for different $\tau_{1}$ values.

It is suggested that in designing engineering structures, decreasing $B i_{1}$ and $B i_{3}$ and increasing $B i_{2}$ will help to increase the thermal shock resistance and the strength against thermal fatigue. In this spirit, trying to modify the effective Biot's numbers remains an efficient way to improve the high temperature performance of structures without changing the physical properties of the material.

\section{Declaration of Competing Interest}

We declare that we have no financial and personal relationships with other people or organizations that can inappropriately influence our work, there is no professional or other personal interest of any nature or kind in any product, service and/or company that could be construed as influencing the position presented in, or the review of, the manuscript entitled, "Biot number effect and non-Fourier effect on temperature field and stress intensity factor of a cracked strip under thermal shock loading".

\section{Acknowledgements}

This work was supported by the National Natural Science Foundation of China (11972041, 11875001), the Strategic Priority Research Program of the Chinese Academy of Sciences (XDB22040102) and the National Key Research and Development Program of China (2016YFA0501601). 


\section{Appendix}

The relations between $B_{i}^{H}$ and $C_{i}^{H}(i=1, \cdots, 8)$ in (40) are:

$$
\begin{cases}B_{1}^{H}=\frac{\mathrm{i}\left(|\xi| C_{1}^{H}+\kappa C_{2}^{H}\right)}{\xi}, & B_{2}^{H}=\mathrm{i} C_{2}^{H} \operatorname{sign}(\xi), \\ B_{3}^{H}=\frac{\mathrm{i}\left(\kappa C_{4}^{H}-|\xi| C_{3}^{H}\right)}{\xi}, & B_{4}^{H}=-\mathrm{i} C_{4}^{H} \operatorname{sign}(\xi), \\ B_{5}^{H}=\frac{\mathrm{i}\left(|\xi| C_{5}^{H}+\kappa C_{6}^{H}\right)}{\xi}, & B_{6}^{H}=\mathrm{i} C_{6}^{H} \operatorname{sgn}(\xi), \\ B_{7}^{H}=\frac{\mathrm{i}\left(\kappa C_{8}^{H}-|\xi| C_{7}^{H}\right)}{\xi}, & B_{8}^{H}=-\mathrm{i} C_{8}^{H} \operatorname{sgn}(\xi)\end{cases}
$$

where $\operatorname{sgn}(\xi)$ is the signum function.

The functions $\bar{u}_{i, \phi}^{P}, \bar{u}_{i, \delta}^{P}, \bar{v}_{i, \phi}^{P}, \bar{v}_{i, \delta}^{P}(i=1,2)$ in (40) are defined as:

$$
\begin{aligned}
& \bar{u}_{1, \phi}^{P}=\frac{-2 \mathrm{e}^{-m y}\left(\left(B i_{2}+m\right) \mathrm{e}^{2 m l_{b}}+B i_{2}-m\right)\left(\left(B i_{1}+m\right) \mathrm{e}^{2 m\left(l_{a}+y\right)}-B i_{1}+m\right) \tilde{\phi}}{(\kappa+1)\left(m^{2}-\xi^{2}\right)\left(\left(B i_{1}+m\right)\left(B i_{2}+m\right) \mathrm{e}^{2 m\left(l_{a}+l_{b}\right)}-\left(m-B i_{1}\right)\left(m-B i_{2}\right)\right)} \\
& \bar{u}_{2, \phi}^{P}=\frac{2 \mathrm{e}^{-m y}\left(\left(B i_{1}+m\right) \mathrm{e}^{2 m l_{a}}+B i_{1}-m\right)\left(\left(B i_{2}+m\right) \mathrm{e}^{2 m l_{b}}+\left(m-B i_{2}\right) \mathrm{e}^{2 m y}\right)}{(\kappa+1)\left(m^{2}-\xi^{2}\right)\left(\left(B i_{1}+m\right)\left(B i_{2}+m\right) \mathrm{e}^{2 m\left(l_{a}+l_{b}\right)}-\left(m-B i_{1}\right)\left(m-B i_{2}\right)\right)} \\
& \bar{v}_{1, \phi}^{P}=\frac{2 \mathrm{i} m \mathrm{e}^{-m y}\left(\left(B i_{2}+m\right) \mathrm{e}^{2 m l_{b}}+B i_{2}-m\right)\left(\left(B i_{1}+m\right) \mathrm{e}^{2 m\left(l_{a}+y\right)}+B i_{1}-m\right)}{(\kappa+1) \xi\left(m^{2}-\xi^{2}\right)\left(\left(B i_{1}+m\right)\left(B i_{2}+m\right) \mathrm{e}^{2 m\left(l_{a}+l_{b}\right)}-\left(m-B i_{1}\right)\left(m-B i_{2}\right)\right)} \\
& \bar{v}_{2, \phi}^{P}=\frac{8 \mathrm{i} m \mathrm{e}^{m\left(l_{a}+l_{b}\right)}\left(B i_{1} \cosh \left(m l_{a}\right)+m \sinh \left(m l_{a}\right)\right)\left(B i_{2} \cosh \left(m\left(y-l_{b}\right)\right)-m \sinh \left(m\left(y-l_{b}\right)\right)\right)}{(\kappa+1) \xi\left(m^{2}-\xi^{2}\right)\left(\left(B i_{1}+m\right)\left(B i_{2}+m\right) \mathrm{e}^{2 m\left(l_{a}+l_{b}\right)}-\left(m-B i_{1}\right)\left(m-B i_{2}\right)\right)} \\
& \bar{u}_{1, \delta}^{P}=0 \\
& \bar{u}_{2, \delta}^{P}=0 \\
& \bar{v}_{1, \delta}^{P}=\frac{4 B i_{1} m_{0} \mathrm{e}^{m_{0}\left(l_{a}-y\right)}\left(\left(m_{0}-B i_{2}\right) \mathrm{e}^{2 m_{0} y}-\left(B i_{2}+m_{0}\right) \mathrm{e}^{\left.2 m_{0} l_{b}\right)}\right.}{(\kappa+1) p m_{0}^{2}\left(\left(B i_{1}+m_{0}\right)\left(B i_{2}+m_{0}\right) \mathrm{e}^{2 m_{0}\left(l_{a}+l_{b}\right)}+\left(B i_{1}-m_{0}\right)\left(m_{0}-B i_{2}\right)\right)} \\
& \bar{v}_{1, \delta}^{P}=\frac{4 B i_{1} m \mathrm{e}^{m_{0} l_{a}-m_{0} y}\left(\left(m_{0}-B i_{2}\right) \mathrm{e}^{2 m_{0} y}-\left(B i_{2}+m_{0}\right) \mathrm{e}^{2 m_{0} l_{b}}\right)}{(\kappa+1) p m_{0}^{2}\left(\left(B i_{1}+m_{0}\right)\left(B i_{2}+m_{0}\right) \mathrm{e}^{2 m_{0}\left(l_{a}+l_{b}\right)}+\left(B i_{1}-m_{0}\right)\left(m_{0}-B i_{2}\right)\right)}
\end{aligned}
$$

A and $\gamma$ are in (44) are defined as:

$$
\mathbf{A}=\left[\begin{array}{cccccccc}
a_{1,1} & a_{1,2} & a_{1,3} & a_{1,4} & 0 & 0 & 0 & 0 \\
0 & 0 & 0 & 0 & a_{2,5} & a_{2,6} & a_{2,7} & a_{2,8} \\
a_{3,1} & a_{3,2} & a_{3,3} & a_{3,4} & a_{3,5} & a_{3,6} & a_{3,7} & a_{3,8} \\
a_{4,1} & a_{4,2} & a_{4,3} & a_{4,4} & a_{4,5} & a_{4,6} & a_{4,7} & a_{4,8} \\
0 & 0 & 0 & 0 & a_{5,5} & a_{5,6} & a_{5,7} & a_{5,8} \\
0 & 0 & 0 & 0 & a_{6,5} & a_{6,6} & a_{6,7} & a_{6,8} \\
-1 & -1 & -1 & -1 & 1 & 1 & 1 & 1 \\
-B_{1} & -B_{2} & -B_{3} & -B_{4} & B_{1} & B_{2} & B_{3} & B_{4}
\end{array}\right]^{-1}
$$

where

$$
\begin{cases}a_{1, j}=\left(\mathrm{i} \xi \nu+B_{j} \lambda_{j}\right) \mathrm{e}^{-\lambda_{j} l_{a}} & j=1, \cdots, 4 \\ a_{2 j}=\left(\mathrm{i} \xi B_{j}+\lambda_{j}\right) \mathrm{e}^{-\lambda_{j} l_{a}} & j=1, \cdots, 4 \\ a_{3, j}=(-1)^{H(j-4.5)}\left(\mathrm{i} \xi \nu+B_{j} \lambda_{j}\right) & j=1, \cdots, 8 \\ a_{4, j}=(-1)^{H(j-4.5)}\left(\mathrm{i} \xi B_{j}+\lambda_{j}\right) & j=1, \cdots, 8 \\ a_{5, j}=\left(\mathrm{i} \xi \nu+B_{j} \lambda_{j}\right) \mathrm{e}^{\lambda_{j} l_{b}} & j=5, \cdots, 8 \\ a_{6, j}=\left(\mathrm{i} \xi B_{j}+\lambda_{j}\right) \mathrm{e}^{\lambda_{j} l_{b}} & j=5, \cdots, 8\end{cases}
$$




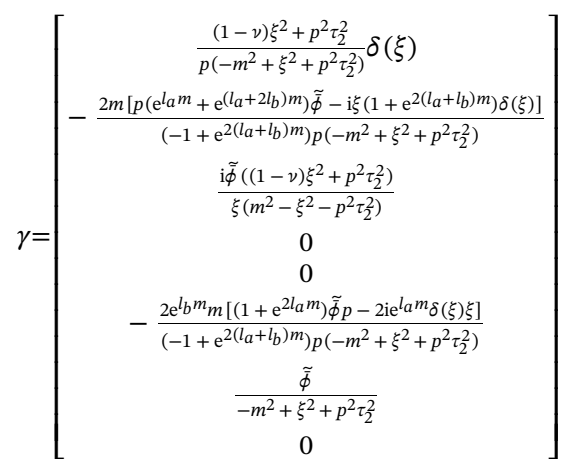

where $H(x)$ in (A.11) is the Heaviside step function. It is a discontinuous function whose value is zero for negative argument and one for positive argument:

$$
H(x)= \begin{cases}0 & x<0 \\ 1 & x>0\end{cases}
$$

$K_{k, l}(k=1,2 ; l=1,2,3)$ in $(48)$ are written as:

$$
\begin{aligned}
& K_{1,1}=\frac{\sin ((x-\eta) \xi)}{2 \sinh ^{2}\left(\xi\left(l_{a}+l_{b}\right)\right)-2 \xi^{2}\left(l_{a}+l_{b}\right)^{2}}\left[4 \xi l_{a}\left(\xi l_{b}\left(\xi l_{b}-1\right)-\sinh ^{2}\left(\xi l_{b}\right)\right)\right. \\
& +2 \xi\left(l_{b}\left(\xi l_{b}\left(\sinh \left(2 \xi l_{a}\right)-1\right)-2 \sinh ^{2}\left(\xi l_{a}\right)\right)+\xi l_{a}^{2}\left(2 \xi l_{b}+\sinh \left(2 \xi l_{b}\right)-1\right)\right) \\
& \left.-\sinh \left(2 \xi\left(l_{a}+l_{b}\right)\right)+\cosh \left(2 \xi\left(l_{a}+l_{b}\right)\right)+\sinh \left(2 \xi l_{a}\right)+\sinh \left(2 \xi l_{b}\right)-1\right] \\
& K_{1,2}=\frac{2 \xi^{2}\left(l_{a}^{2} \sinh ^{2}\left(\xi l_{b}\right)-l_{b}^{2} \sinh ^{2}\left(\xi l_{a}\right)\right)}{\xi^{2}\left(l_{a}+l_{b}\right)^{2}-\sinh ^{2}\left(\xi\left(l_{a}+l_{b}\right)\right)} \cos ((x-\eta) \xi) \\
& K_{1,3}=-\frac{2 \pi \sin (\xi x)}{n}\left[\frac{1}{4}(\kappa+1)\left(2|\xi|\left(\mathbf{A}_{1}-\mathbf{A}_{3}\right) \cdot \mathbf{B}+(\kappa-1)\left(\mathbf{A}_{2}+\mathbf{A}_{4}\right) \cdot \mathbf{B}\right)\right. \\
& \left.+\frac{m\left(\left(B i_{1}+m\right) \mathrm{e}^{2 m l_{a}}+B i_{1}-m\right)\left(\left(B i_{2}+m\right) \mathrm{e}^{2 m l_{b}}+B i_{2}-m\right)}{(m-\xi)(m+\xi)\left(\left(B i_{1}+m\right)\left(B i_{2}+m\right) \mathrm{e}^{2 m\left(l_{a}+l_{b}\right)}-\left(m-B i_{1}\right)\left(m-B i_{2}\right)\right)}\right] \\
& K_{2,1}=\frac{2 \xi^{2}\left(l_{b}^{2} \sinh ^{2}\left(\xi l_{a}\right)-l_{a}^{2} \sinh ^{2}\left(\xi l_{b}\right)\right)}{\xi^{2}\left(l_{a}+l_{b}\right)^{2}-\sinh ^{2}\left(\xi\left(l_{a}+l_{b}\right)\right)} \cos ((x-\eta) \xi) \\
& K_{2,2}=\frac{\sin ((x-\eta) \xi)}{2 \sinh ^{2}\left(\xi\left(l_{a}+l_{b}\right)\right)-2 \xi^{2}\left(l_{a}+l_{b}\right)^{2}}\left[2 \xi l_{a}\left(2 \sinh ^{2}\left(\xi l_{b}\right)-2 \xi l_{b}\left(\xi l_{b}+1\right)\right)\right. \\
& +2 \xi\left(l_{b}\left(\xi l_{b}\left(\sinh \left(2 \xi l_{a}\right)-1\right)+2 \sinh ^{2}\left(\xi l_{a}\right)\right)+\xi l_{a}^{2}\left(-2 \xi l_{b}+\sinh \left(2 \xi l_{b}\right)-1\right)\right) \\
& \left.\left.-\sinh \left(2 \xi\left(l_{a}+l_{b}\right)\right)+\cosh \left(2 \xi\left(l_{a}+l_{b}\right)\right)+\sinh \left(2 \xi l_{a}\right)+\sinh \left(2 \xi l_{b}\right)-1\right)\right] \\
& K_{2,3}=\frac{1}{4|\xi|} \mathrm{i}(\kappa+1) \xi\left(2|\xi|\left(\mathbf{A}_{1}+\mathbf{A}_{3}\right) \cdot \mathbf{B}+(\kappa+1)\left(\mathbf{A}_{2}-\mathbf{A}_{4}\right) \cdot \mathbf{B}\right)+ \\
& \frac{4 i \xi \mathrm{e}^{m\left(l_{a}+l_{b}\right)}\left(B i_{1} \sinh \left(m l_{a}\right)+m \cosh \left(m l_{a}\right)\right)\left(B i_{2} \cosh \left(m l_{b}\right)+m \sinh \left(m l_{b}\right)\right)}{(m-\xi)(m+\xi)\left(\left(m-B i_{1}\right)\left(m-B i_{2}\right)-\left(B i_{1}+m\right)\left(B i_{2}+m\right) \mathrm{e}^{2 m\left(l_{a}+l_{b}\right)}\right)}
\end{aligned}
$$

where the subscript $i(i=1,2,3,4)$ of A denotes the ith column of $\mathbf{A}$ and the operator "." denotes dot product of vectors.

\section{References}

[1] Liu YX, Wu XF, Guo QK, Jiang CP, Song F, Li J. Experiments and numerical simulations of thermal shock crack patterns in thin circular ceramic specimens. Ceram Int 2015;41:1107-14.

[2] Rendtorff NM, Garrido LB, Aglietti EF. Thermal shock resistance and fatigue of zircon-mullite composite materials. Ceram Int 2011;37:1427-34.

[3] Han JC. Thermal shock resistance of ceramic coatings. Acta Mater 2007;55:3573-81.

[4] Lu TJ, Fleck NA. The thermal shock resistance of solids. Acta Mater 1998;46:4755-68.

[5] Choi YK, Jeon S, Jeon MS, Shin HG, Chun HH, Lee YS, et al. Crack propagation behavior of TiN coatings by laser thermal shock experiments. Appl Surf Sci 2012;258:8752-7.

[6] Mauer G, Vassen R. Current developments and challenges in thermal barrier coatings. Surf Engng 2011;27:477-9.

[7] Li J, Song F, Jiang CP. Direct numerical simulations on crack formation in ceramic materials under thermal shock by using a non-local fracture model. J Eur Ceram Soc 2013;33:2677-87.

[8] Li J, Song F, Jiang CP. A non-local approach to crack process modeling in ceramic materials subjected to thermal shock. Engng Fract Mech 2015;133:85-98.

[9] Song F, Meng SH, Xu XH, Shao YF. Enhanced thermal shock resistance of ceramics through biomimetically inspired nanofins. Phys Rev Lett 2010;104:125502.

[10] Fu JW, Chen ZT, Qian LF, Xu YD. Non-fourier thermoelastic behavior of a hollow cylinder with an embedded or edge circumferential crack. Engng Fract Mech 2014;128:103-20.

[11] Cattaneo C. Sulla conduzione del calore. Atti Sem Mat Fis Univ Modena 1948.

[12] Cattaneo C. A form of heat conduction equation which eliminates the paradox of instantaneous propagation. Compte Rendus 1958;247:431-3.

[13] Vernotte P. Les paradoxes de la théorie continue de léquation de la chaleur. CR Hebd Seances Acad Sci 1958;246:3154-5. 
[14] Joseph DD, Preziosi L. Heat waves. Rev Modern Phys 1989;61:41-73.

[15] ÖZisik MN, Tzou DY. On the Wave Theory in Heat Conduction. J Heat Transfer 1994;116:526-35.

[16] Tzou DY, Transfer M. The generalized lagging response in small-scale and high-rate heating. Int J Heat Mass Transf 1995;38:3231-40.

[17] Tzou DY. Macro-to microscale heat transfer: the lagging behavior. John Wiley \& Sons; 2014.

[18] Alvarez FX, Jou D. Memory and nonlocal effects in heat transport: From diffusive to ballistic regimes. Appl Phys Lett 2007;90(8).

[19] Chen ZT, Hu KQ. Thermo-elastic analysis of a cracked half-plane under a thermal shock impact using the hyperbolic heat conduction theory. J Therm Stresses 2012;35:342-62.

[20] Chen ZT, Hu KQ. Hyperbolic heat conduction in a cracked thermoelastic half-plane bonded to a coating. Int J Thermophys 2012;33:895-912.

[21] Guo SL, Wang BL. Thermal shock cracking behavior of a cylinder specimen with an internal penny-shaped crack based on non-fourier heat conduction. Int $J$ Thermophys 2016;37:17.

[22] Atkinson C, Craster RV. Fracture in fully coupled dynamic thermoelasticity. J Mech Phys Solids 1992;40:1415-32.

[23] Fu JW, Chen ZT, Qian LF, Xu YD. Thermal fracture of cracked cylinders associated with nonclassical heat conduction: the effect of material property. J Therm Stresses 2016;39:1119-37.

[24] Li W, Song F, Li J, Abdelmoula R, Jiang C-P. Non-fourier effect and inertia effect analysis of a strip with an induced crack under thermal shock loading. Engng Fract Mech 2016;162:309-23.

[25] Li W. Thermal Barrier Effect, Non-Fourier Effect and Inertia Effect on a Cracked Plate under Thermal Shock Loading Villetaneuse: Université Paris 13; 2016.

[26] Kaminski W. Hyperbolic heat conduction equation for materials with a nonhomogeneous inner structure. J Heat Transfer 1990;112:555-60.

[27] Antaki PJ. New interpretation of non-Fourier heat conduction in processed meat. J Heat Transfer 2005;127:189-93.

[28] Guo SL, Wang BL, Wang KF. Dual-phase-lag heat conduction and associate fracture mechanics of a ceramic fiber/matrix composite cylinder. Ceram Int 2019;45:4707-17.

[29] Graßmann A, Peters F. Experimental investigation of heat conduction in wet sand. Heat Mass Transf 1999;35:289-94.

[30] Roetzel W, Putra N, Das SK. Experiment and analysis for non-Fourier conduction in materials with non-homogeneous inner structure. Int J Therm Sci 2003;42:541-52.

[31] Keles I, Conker C. Transient hyperbolic heat conduction in thick-walled FGM cylinders and spheres with exponentially-varying properties. Eur J Mech A Solids 2011;30:449-55.

[32] Li W, Li J, Abdelmoula R, Song F, Jiang CP. Inertia effect analysis of a half-plane with an induced crack under thermal loading using hyperbolic heat conduction. ZAMM - Appl Math Mec 2015;96:939-55.

[33] Jeffrey A, Zwillinger D. Table of Integrals, Series, and Products. Academic Press; 2007.

[34] Miller MK, Guy J, W. T. Numerical inversion of the Laplace transform by use of Jacobi polynomials. SIAM J Numer Anal 1966;3:624-35.

[35] Muskhelishvili NI, Radok JRM. Singular Integral Equations: Boundary Problems of Function Theory and Their Application to Mathematical Physics. 2nd ed. Courier Corporation; 1953.

[36] Delale F, Erdogan F. The crack problem for a nonhomogeneous plane. J Appl Mech 1983;50:609-14.

[37] Erdogan F, Wu BH. The surface crack problem for a plate with functionally graded properties. J Appl Mech 1997;64:449-56.

[38] Hu KQ, Chen ZT. Thermoelastic analysis of a partially insulated crack in a strip under thermal impact loading using the hyperbolic heat conduction theory. Int $J$ Engng Sci 2012;51:144-60. 